\title{
La réceptivité des sols aux fusarioses vasculaires: rapports entre résistance et microflore autochtone avec référence particulière aux Fusarium non pathogènes $\left(^{*}\right)$
}

\author{
G Tamietti ** R Pramotton \\ Istituto di Patologia vegetale. Università di Torino, Via Pietro Giuria 15, 10126 Turin, Italie
}

(Reçu le 20 juillet 1989 ; accepté le 13 octobre 1989)

\begin{abstract}
Résumé - L'estimation du niveau de réceptivité aux fusarioses vasculaires de 4 échantillons de sols provenant de l'Italie du Nord a permis de montrer que 3 d'entre eux sont résistants à la fusariose du lin, le quatrième étant beaucoup plus sensible. Dans tous les cas, le traitement thermique à la vapeur détruit la résistance de ces sols qui est donc de nature microbiologique. L'isolement d'une collection de souches de Fusarium spp à partir de ces 4 échantillons de sols et leur introduction dans un sol traité à la chaleur montrent qu'une forte proportion de souches de $F$ oxysporum est capable d'induire la résistance dans ce sol désinfecté. Le rôle de $F$ oxysporum dans les mécanismes de résistance apparaît plus important que celui des $F$ solani et des $F$ roseum. L'aptitude des souches de $F$ oxysporum à diminuer l'incidence de la maladie se manifeste de manière similaire dans les différents types de sols traités à la chaleur. Cette aptitude n'est pas corrélée à la capacité des souches à se multiplier saprophytiquement dans ces sols.
\end{abstract}

sol / fusariose vasculaire / résistance / microflore autochtone

Summary - Soil-suppressiveness to Fusarium-wilt: relationship between suppressiveness and indigenous microflora of the soil with special emphasis on the non-pathogenic Fusarium. The microbiological nature of the suppressiveness to Fusarium-wilt was established by comparative research carried out in Northern Italy, on 4 different soil samples collected whose physico-chemical and cultural features are summarized in table I. Both microbial populations (table II) and suppressiveness to Fusarium-wilt of flax (fig 1) is the soils from Albenga, Carmagnola and Santena were at higher levels than those in the soil from Donnas.

A large proportion of the strains of Fusarium spp isolated from these soils were able to induce suppressiveness to Fusarium-wilt of flax in steamed soil. Among the non-pathogenic Fusaria, $F$ oxysporum appeared to be more involved than $\mathrm{F}$ solani and $\mathrm{F}$ roseum in the mechanisms of the suppression (fig 2). Soil suppressiveness was conditioned not only by the density but also by the composition of the population of non-pathogenic $\mathrm{F}$ oxysporum present in each soil (fig 3). There was no relationship between the ability of non-pathogenic strains of $\mathrm{F}$ oxysporum to colonize the steamed soil (table III) and to induce suppressiveness (table IV, V). Moreover the activity of non-pathogenic strains of $\mathrm{F}$ oxysporum was not influenced by the physical and chemical characteristics of the tested soil samples.

soil / Fusarium-wilt / resistance / indigenous microflora

\footnotetext{
* Gruppo di Ricerca per la Patologia delle Piante ortensi. Pubblicazione $n^{\circ} 241$.

* Correspondance et tirés à part.
} 


\section{INTRODUCTION}

La résistance du sol, définie comme l'aptitude d'un sol à réduire l'incidence et/ou la gravité de maladies des plantes provoquées par des agents pathogènes d'origine tellurgique naturellement présents ou artificiellement introduits dans le sol, a été mise en évidence dans le cas de maladies provoquées par Gaeumannomyces graminis (Sacc) v Arx et Olivier (Gerlagh, 1968), Pythium ultimum Trow (Bouhot, 1975), Phytophthora cinnamomi Rands (Baker, 1978), Rhizoctonia solani Kühn (Henis et al, 1978), Pythium splendens Braun (Ko et Ho, 1983) et certaines formes pathogènes de Fusarium oxysporum Schlecht.

De nombreuses recherches consacrées aux mécanismes de résistance aux fusarioses ont mis en évidence la nature microbiologique du phénomène qui peut être mis en relation avec la présence dans le sol de bactéries fluorescentes productrices de sidérophores, Pseudomonas putida et $P$ fluorescens en particulier (Scher et Baker, 1980, 1982 ; Kloepper et al, 1980) ou de Fusarium spp, en particulier $F$ oxysporum non pathogènes, (Rouxel et al, 1979; Ogawa et Komada, 1984 ; Schneider, 1984 ; Garibaldi et al, 1985 ; Tamietti et Alabouvette, 1986). Bien que des microorganismes différents puissent être actifs en même temps, l'appréciation du rôle de chacun d'eux dans le phénomène de résistance et la connaissance de leurs exigences écologiques et de leurs modalités d'action sont très importantes en vue de l'utilisation de ces microorganismes pour la lutte microbiologique.

L'expérimentation rapportée dans cet article avait pour but de déterminer les microorganismes responsables des différences de niveaux de résistance aux fusarioses vasculaires observées dans 4 sols provenant de 4 localités différentes de l'Italie du Nord, d'analyser le rôle joué par les espèces de Fusarium les plus fréquemment isolées de ces sols et d'établir une relation entre la capacité de ces souches à coloniser les sols et leur aptitude à rendre les sols résistants.

\section{MATÉRIEL ET MÉTHODES}

\section{Sols et analyses microbiologiques}

Les recherches portent sur 4 sols agricoles provenant de Albenga (Savone), Donnas (Aoste), Carmagnola (Turin), Santena (Turin) dont les caractéristiques physico-chimiques et culturales sont résumées dans le tableau I. Les échantillons de sol sont obtenus en mélangeant soigneusement 5 sub-échantillons prélevés à des points différents d'une même exploitation dans chaque localité, dans l'horizon $0-20 \mathrm{~cm}$ de profondeur.

Tableau I. Caractéristiques physico-chimiques et agronomiques des échantillons de terre utilisés au cours de cette recherche.

\begin{tabular}{|c|c|c|c|c|c|}
\hline & & & & Sols & \\
\hline & & Albenga & Carmagnola & Donnas & Santena \\
\hline Sable & $(\%)$ & 58 & 34 & 49 & 79 \\
\hline Limon grossier & $(\%)$ & 14 & 33 & 27 & 10 \\
\hline Limon fin & $(\%)$ & 15 & 24 & 18 & 5 \\
\hline Argile & (\%) & 13 & 9 & 6 & 6 \\
\hline $\mathrm{pH}$ eau & & 7,0 & 7,5 & 7,1 & 6,7 \\
\hline Matière organiques & $(\%)$ & 2,33 & 1,53 & 5,58 & 1,19 \\
\hline C organique & $(\%)$ & 1,35 & 0,89 & 3,24 & 0,69 \\
\hline$N$ total & $(\%)$ & 1,34 & 0,97 & 2,62 & 0,80 \\
\hline $\mathrm{C} / \mathrm{N}$ & & 10,1 & 9,2 & 12,4 & 8,6 \\
\hline Capacité d'échange & $\mathrm{M} \mathrm{eq} / 100 \mathrm{~g}$ & 7,93 & 6,31 & 17 & 4,78 \\
\hline $\mathrm{Ca}$ échangeable & $M$ eq/100 g & 12,29 & 9,45 & 18,84 & 4,43 \\
\hline Mg échangeable & $\mathrm{M} \mathrm{eq} / 100 \mathrm{~g}$ & 1,57 & 0,88 & 3,75 & 1,20 \\
\hline K échangeable & $\mathrm{M} \mathrm{eq} / 100 \mathrm{~g}$ & 1,18 & 0,34 & 0,59 & 0,57 \\
\hline P échangeable & $\mathrm{ppm}$ & 173 & 36 & 321 & 137 \\
\hline Fe échangeable EDTA & ppm & 327 & 231 & 546 & 526 \\
\hline Mn échangeable EDTA & ppm & 231 & 358 & 305 & 145 \\
\hline Zn échangeable EDTA & ppm & 57,3 & 8,1 & 150 & 35,4 \\
\hline Cu échangeable EDTA & ppm & 48,5 & 9,9 & 87,4 & 8,8 \\
\hline Cultures pratiquées & & Légumes & Légumes & $\begin{array}{l}\text { Légumes/ } \\
\text { pomme de terre }\end{array}$ & $\begin{array}{l}\text { Légumes/ } \\
\text { maïs }\end{array}$ \\
\hline
\end{tabular}


Les sols sont utilisés tels quels ou après désinfection à la vapeur $\left(100^{\circ} \mathrm{C}, 60 \mathrm{~min}\right)$.

Les analyses de la microflore fongique sont réalisées par la méthode des suspensions-dilutions. Une série de suspensions-dilutions au 1/10 est réalisée à partir d'un échantillon de $10 \mathrm{~g}$ de sol mis en suspension dans $90 \mathrm{ml}$ d'eau stérile. Un $\mathrm{ml}$ de la suspension diluée est incorporé à $9 \mathrm{ml}$ de milieu de culture : extrait de malt gélosé acidifié à l'acide citrique (AMEA) pour la microflore totale, milieu de Komada (1975) pour les Fusarium. Les bactéries et les actinomycètes sont dénombrées par étalement de $20 \mu \mathrm{l}$ des mêmes suspensions-dilutions en boîtes de Petri contenant $10 \mathrm{ml}$ du milieu B de King et al (1954).

Pour chaque analyse, 2 suspensions-dilutions sont réalisées à partir d'échantillons différents, 10 boîtes de Petri sont ensemencées pour chaque dilution dans le cas des champignons, 5 dans le cas des bactéries. Au cours de cette expérimentation, les analyses microbiologiques ont été réalisées 6 fois à partir d'échantillons de chaque sol prélevés à des périodes différentes de l'année. Les résultats présentés correspondent à la moyenne des densités de populations enregistrées au cours de ces 6 analyses.

\section{Évaluation du niveau de résistance des sols}

Des échantillons de chacun des 4 sols désinfectés ou non sont infestés avec des doses croissantes $(0,5,1$, 2,4 et $8 \times 10^{3} \mathrm{cfu} / \mathrm{ml}$ ) d'un inoculum de $F$ oxysporum f sp lini produit selon la technique décrite par TelloMarquina et Alabouvette (1984). La terre est répartie en pots de $500 \mathrm{ml}$, chaque traitement comporte 6 pots semés avec 12 graines de lin (Linum usitatissimum $L$ ) cv Hera. Les plantules sont cultivées en serre à la température minimale de $19-20^{\circ} \mathrm{C}$ et photopériode minimale de 12 h jusqu'à la fin de leur floraison (80-90 j).

La gravité de la maladie est évaluée par dénombrement hebdomadaire des plantes malades qui sont éliminées. Le niveau de résistance du sol est exprimé par le pourcentage de plantes saines.

A l'issue de la première culture, un second semis de lin est effectué dans les mêmes sols, sans nouvel apport de l'agent pathogène. La gravité de la maladie est évaluée dans les mêmes conditions que précédemment.

\section{Aptitude des Fusarium à induire la résistance dans les sols désinfectés}

Quatre-vingt-dix souches de $F$ oxysporum, 28 souches de $F$ roseum et 27 souches de $F$ solani ont été choisies au hasard parmi les colonies de Fusarium spp isolées sur milieu de Komada à partir des 4 sols étudiés. Les souches ont été comparées pour leur aptitude à rétablir la résistance à la fusariose du lin dans un autre échantillon de sol préalablement traité à la chaleur.

Deux $\mathrm{ml}$ d'une suspension conidienne obtenue par lavage d'une culture sur AMEA âgée de 8 j sont incorporés dans 1,5 I de sol d'Albenga désinfecté. Afin de permettre aux Fusarium spp de coloniser intensément le sol, celui-ci est incubé pendant 20 j à $23-25^{\circ} \mathrm{C}$, avant d'être infesté par apport de $3 \times 10^{3} \mathrm{cfu} / \mathrm{ml}$ de l'agent pathogène $F$ oxysporum $f$ sp lini. L'échantillon est réparti en 3 pots de $500 \mathrm{ml}$, immédiatement semés avec du lin, variété Hera. La culture et l'évaluation de la gravité de la maladie sont réalisées dans les conditions décrites précédemment.

Dans une autre expérimentation, 16 souches de $F$ oxysporum ont été utilisées pour infester les 4 sols d'Albenga, Carmagnola, Santena, Donnas préalablement traités à la vapeur à $100^{\circ} \mathrm{C}$ pendant $60 \mathrm{~min}$. L'infestation et l'incubation des sols sont réalisées comme il est décrit ci-dessus. A l'issue de la période d'incubation, la densité des populations de Fusarium est déterminée par la méthode des suspensions-dilutions et mise en culture sur AMEA, dans les conditions décrites précédemment.

Les sols précolonisés sont ensuite infestés par l'agent pathogène à la concentration de $3 \times 10^{3} \mathrm{cfu} / \mathrm{ml}$, et la résistance des sols est évaluée par culture du lin, comme décrit précédemment (6 répétitions de 10 plantes par traitement).

Cette expérimentation a été répétée une seconde fois dans des conditions analogues.

Les résultats expérimentaux ont été comparés par analyse de la variance suivie du test de Duncan, après transformation angulaire pour le pourcentage de plantes saines.

\section{RÉSULTATS}

\section{Comparaison des niveaux de résistance des sols à la fusariose du lin}

La figure 1 indique que les sols d'Albenga, Carmagnola et Santena montrent un comportement analogue vis-à-vis de la fusariose vasculaire du lin.

Ils présentent un niveau de résistance élevé ; le pourcentage de plantes saines est très important et ne varie pas significativement avec l'aug-

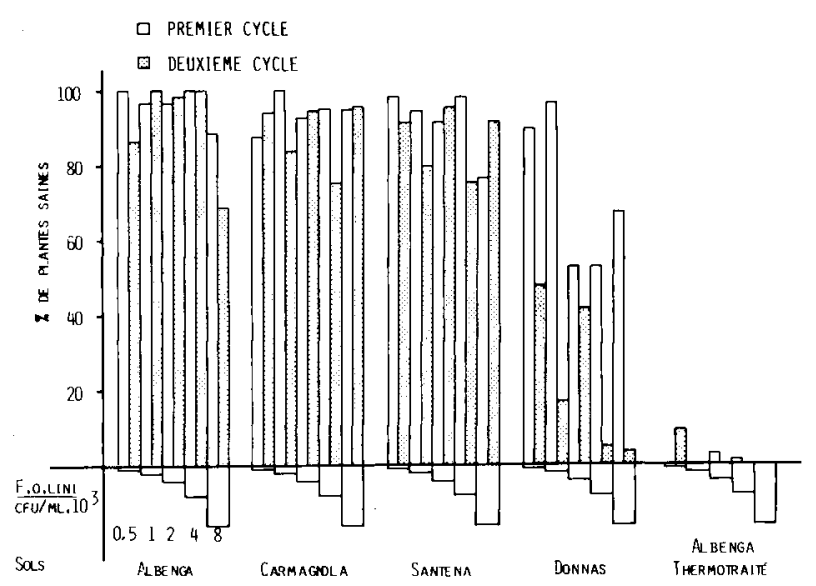

Fig. 1. Niveau de réceptivité de 4 échantillons de sols et de celui d'Albenga traité à la chaleur à la fusariose vasculaire du lin à l'issue de la première culture effectuées dans les mêmes échantillons de sols infestés avec des doses croissantes de $F$ oxysporum f sp lini : pourcentages de plantes saines $80 \mathrm{j}$ après le semis. 
mentation de la densité d'inoculum de $0,5 \times 10^{3}$ à $8 \times 10^{3} \mathrm{cfu} / \mathrm{ml}$ de $F$ oxysporum $\mathrm{f} \mathrm{sp}$ lini. De plus, cette résistance apparaît stable, le pourcentage de plantes saines, à l'issue du deuxième cycle cultural n'est pas différent de celui enregistré au terme du premier cycle.

Ce niveau de résistance élevé et stable s'est manifesté de manière analogue au cours de 3 expérimentations réalisées durant 2 années consécutives.

Le sol de Donnas se révèle beaucoup plus sensible à la fusariose du lin que les 3 sols précédents. Le pourcentage de plantes saines diminue proportionnellement à l'augmentation de la densité d'inoculum de l'agent pathogène et la maladie se manifeste plus intensément au cours du deuxième cycle cultural qu'au cours du premier (fig 1).

Le traitement thermique à $100{ }^{\circ} \mathrm{C}$ pendant $60 \mathrm{~min}$ a détruit la résistance du sol d'Albenga (fig 1), le pourcentage de plantes mortes dépasse $90 \%$ pour la dose d'inoculum la plus faible $\left(0,5 \times 10^{3} \mathrm{cfu} / \mathrm{ml}\right)$. Ce résultat a été confirmé pour les 3 autres sols étudiés (tableaux IV et V).

\section{Analyse des populations microbiennes des sols}

Le tableau II indique la densité des populations microbiennes analysées dans les 4 sols étudiés. Le dénombrement des populations bactériennes totales réalisé sur le milieu $B$ de King montre que le sol sensible de Donnas est celui qui possède la microflore bactérienne la plus pauvre. Les sols d'Albenga et Carmagnola présentent des niveaux de populations 3 fois plus élevés, celui de Santena étant intermédiaire.

II en est de même pour les populations de Pseudomonas fluorescents, le sol de Donnas est le plus pauvre, celui de Carmagnola est le plus riche.

Tableau II. Densité des populations microbiennes (cfu/g de sol sec) des échantillons de sols d'Albenga, Carmagnola, Donnas et Santena : moyennes de 6 analyses différentes.

(") Les moyennes suivies d'une même lettre ne sont pas significativement différentes au seuil de $5 \%$ d'après le test de Duncan.

\begin{tabular}{|c|c|c|c|c|c|}
\hline \multirow[b]{2}{*}{ Sol } & \multicolumn{2}{|c|}{$\begin{array}{l}\text { Bactéries } \times 10^{6} \\
\text { Totaux fluoresc }\end{array}$} & \multirow{2}{*}{\multicolumn{3}{|c|}{$\begin{array}{c}\text { Champignons } \times 10^{4} \\
\text { Totaux }(*) \text { Fusarium(*) }\end{array}$}} \\
\hline & $\begin{array}{c}\text { Totaux } \\
(*)\end{array}$ & fluoresc & Totaux(*) & & $\lim _{*}^{*}$ \\
\hline $\begin{array}{l}\text { Albenga } \\
\text { Carmagnola } \\
\text { Donnas } \\
\text { Santena }\end{array}$ & 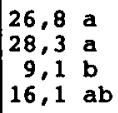 & $\begin{array}{l}0,2 \\
0,5 \\
0,1 \\
0,3\end{array}$ & $\begin{array}{rl}5,2 & \mathrm{bc} \\
13,7 & \mathrm{a} \\
7,6 & \mathrm{c} \\
8,2 & \mathrm{ab}\end{array}$ & $\begin{array}{ll}0,9 & \mathrm{bc} \\
1,1 & \mathrm{ab} \\
0,5 & \mathrm{c} \\
1,4 & \mathrm{a}\end{array}$ & $\begin{array}{r}17,3 \\
8,0 \\
6,6 \\
17,1\end{array}$ \\
\hline
\end{tabular}

En ce qui concerne la mycoflore révélée sur le milieu malt acidifié, le sol d'Albenga est le plus pauvre, suivi par le sol de Donnas, alors que le sol de Carmagnola est de nouveau le plus riche.

Les sols de Santena et Carmagnola sont ceux qui hébergent la population fusarienne la plus importante, celui de Donnas étant de nouveau le plus pauvre.

Si l'on considère l'importance relative de la flore fusarienne au sein de la mycoflore totale, elle représente plus de $17 \%$ dans les sols d'Albenga et Santena, $8 \%$ dans celui de Carmagnola et $6,6 \%$ dans celui de Donnas.

\section{Aptitude des Fusarium spp à induire la résistance dans un sol traité à la chaleur}

Afin d'évaluer l'aptitude des souches de Fusarium spp à induire la résistance dans le sol d'Albenga traité à la vapeur, 5 classes d'activité ont été déterminées en fonction du pourcentage de plantes saines enregistrées $65 \mathrm{j}$ après le semis dans le sol précolonisé par les souches non pathogènes puis infesté par l'agent pathogène.

La figure 2 indique que $66 \%$ des souches de $F$ oxysporum étudiées ont permis d'établir un niveau élevé (classe $\mathbf{8 0 - 1 0 0 \% ~ d e ~ p l a n t e s ~ s a i n e s ) ~}$ de résistance dans le sol traité à la chaleur, alors que $6,5 \%$ des souches se sont révélées inactives (classe $0-20 \%$ de plantes saines).

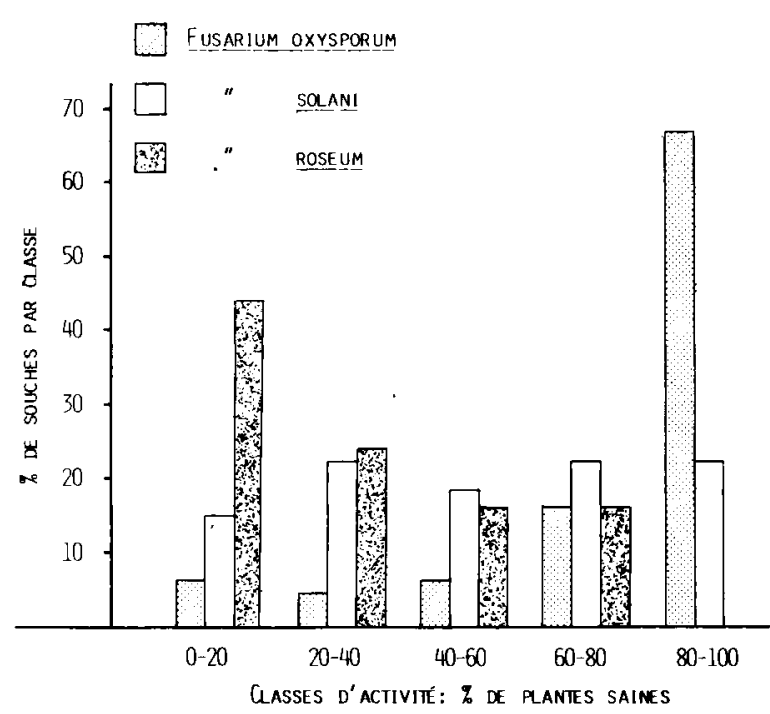

Fig. 2. Aptitude de différentes souches de $F$ oxysporum, $F$ solani et $F$ roseum à rétablir la résistance dans l'échantillon de sol d'Albenga préalablement traité à la chaleur : répartition des souches dans 5 classes d'activité correspondant à $0-20$, $20-40,40-60,60-80$ et $80-100 \%$ de plantes saines de lin 65 j après le semis dans l'échantillon de sol précolonisé par les souches non pahogènes puis infesté par $F$ oxysporum f $s p$ lini a la dose de $3 \times 10^{3} \mathrm{cfu} / \mathrm{ml}$. 
Aucune souche de $F$ roseum ne figure dans la classe correspondant à l'efficacité maximale, tandis que $44 \%$ d'entre elles se sont montrées inactives.

Les souches de $F$ solani sont équitablement réparties dans les différentes classes d'activité ; cette espèce dans l'ensemble a révélé un comportement intermédiaire entre $F$ oxysporum et Froseum.

Quatre-vingt-dix j après le semis, la mortalité est totale dans les sols précolonisés par les souches de $F$ roseum et $F$ solani. Seulement $8 \%$ des souches de $F$ oxysporum sont capables de conférer une résistance durable à l'échantillon d'Albenga traité à la chaleur.

La figure 3 indique la répartition des souches de $F$ oxysporum dans les différentes classes d'efficacité, $90 \mathrm{j}$ après le semis, en fonction du sol d'où elles ont été isolées. Parmi les souches isolées du sol sensible de Donnas, $72 \%$ figurent dans la classe $0-20 \%$ de plantes saines et aucune n'apparaît dans les classes d'efficacité supérieure à $60 \%$ de plantes saines. Au contraire, parmi les souches isolées des sols résistants, 5 à $15 \%$ d'entre elles appartiennent aux classes d'efficacité importante (supérieure à $60 \%$ de plantes saines), en particulier $12 \%$ des souches isolées du sol d'Albenga montrent une efficacité maximale.

Afin d'étudier l'influence du type de sol sur l'aptitude des souches à réduire la gravité de la fusariose du lin, 16 souches de $F$ oxysporum, 10 efficaces et 6 peu efficaces au cours de l'expérimentation précédente ont été choisies pour infes-

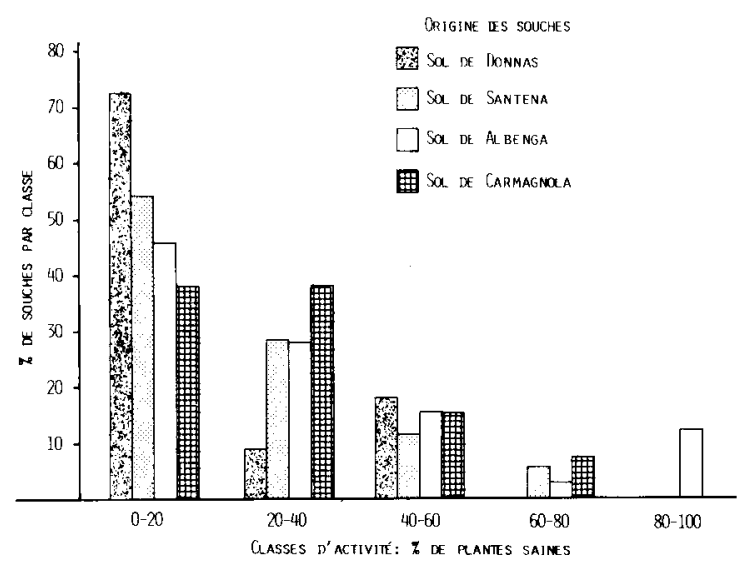

Fig. 3. Aptitude de différentes souches de $F$ oxysporum non pathogènes isolées des échantillons de sols d'Albenga, Carmagnola, Donnas et Santena à rétablir la résistance à la fusariose vasculaire du lin dans l'échantillon d'Albenga traité à la chaleur : répartition des souches dans 5 classes d'activité correspondant à $0-20,20-40,40-60,60-80$ et $80-100 \%$ de plantes saines de lin $90 \mathrm{j}$ après le semis dans le sol précolonisé par les souches non pathogènes puis infesté par $F$ oxysporum f sp lini à la dose de $3 \times 10^{3} \mathrm{cfu} / \mathrm{ml}$.
Tableau III. Densité des populations (cfu/g de sol sec $x 10^{4}$ ) de $F$ oxysporum non pathogènes $20 \mathrm{j}$ après l'apport d'une suspension conidienne dans les échantillons de sols d'Albenga, Carmagnola, Donnas et Santena préalablement traités à la vapeur $\left(100^{\circ} \mathrm{C}\right.$, $60 \mathrm{~min}$ ).

(*) Les valeurs suivies d'un * sont significativement différentes des valeurs correspondantes d'une même ligne au seuil de $5 \%$ d'après le test de Duncan.

$\left({ }^{*}\right)$ Les moyennes suivies d'une même lettre ne sont pas significativement différentes au seuil de $5 \%$ d'après le test de Duncan.

\begin{tabular}{|c|c|c|c|c|c|}
\hline Souches & Albenga & $\begin{array}{c}\text { Sols } \\
\text { |Carmagnola }\end{array}$ & *) Santena & Donnas & $\begin{array}{l}\text { Effet } \\
\text { souches ( }\end{array}$ \\
\hline $\begin{array}{l}3 \\
24 \\
20 a \\
32 \\
8 a \\
22 \\
26 s \\
6 a \\
21 a \\
23 a \\
17 c \\
7 \\
11 v \\
22 s \\
9 a \\
8 s\end{array}$ & $\begin{array}{r}18,8 \\
7,8 \\
2,7 \\
20,5 \\
7,4 \\
7,7 \\
3,8 \\
8,1 \\
6,3 \\
13,6 \\
7,2 \\
2,5 \\
0,3 \\
1,1 \\
1,4 \\
1,8\end{array}$ & $\begin{array}{r}10,7 \\
20,4 \\
9,6 \\
16,4 \\
26,3 \\
13,8 \\
7,5 \\
8,6 \\
14,0 \\
25,9 \\
13,6 \\
0,7 \\
0,8 \\
2,5 \\
1,3 \\
8,4\end{array}$ & $\begin{array}{r}37,3 \\
15,0 \\
11,6 \\
9,1 \\
4,4 \\
6,8 \\
2,2 \\
9,3 \\
0,8 \\
7,4 \\
7,3 \\
2,5 \\
0,4 \\
1,2 \\
1,0 \\
1,3\end{array}$ & $\begin{array}{rl}62,9 & * \\
18,5 & \\
33,0 \\
16,4 \\
4,3 \\
6,9 \\
5,4 \\
7,3 \\
7,1 \\
8,3 \\
3,4 \\
2,4 \\
5,4 \\
1,9 \\
2,2 \\
1,6\end{array}$ & $\begin{array}{rr}32,4 & \mathrm{a} \\
15,4 & \mathrm{~b} \\
10,5 & \mathrm{bcd} \\
15,7 & \mathrm{~b} \\
10,6 & \mathrm{bcd} \\
8,8 & \mathrm{bcd} \\
4,7 & \mathrm{ccd} \\
10,8 & \mathrm{bcd} \\
7,1 & \mathrm{~d} \\
13,8 & \mathrm{bc} \\
7,9 & \mathrm{~d} \\
2,0 & \mathrm{~d} \\
1,7 & \mathrm{~d} \\
1,7 & \mathrm{~d} \\
1,5 & \mathrm{~d} \\
3,2 & \mathrm{~d}\end{array}$ \\
\hline $\begin{array}{l}\text { Effet } \\
\left.\text { sols },{ }^{\circ}\right)\end{array}$ & 6,9 a & 11,9 a & 7,3 a & 11,7 a & \\
\hline
\end{tabular}

ter les 4 sols d'Albenga, Carmagnola, Santena et Donnas préalablement traités à la vapeur.

Le tableau III indique la densité des populations déterminée après la période d'incubation, 20 j après l'apport de la suspension de conidies. II montre que pour une même souche de Fusarium il n'existe pas de différence de colonisation en fonction du type de sol, à deux exceptions près (souche 3 et $8 \mathrm{a}$ ).

Par contre, ces souches de Fusarium montrent une grande diversité quant à leur aptitude à coloniser les sols. En moyenne, la souche la plus performante atteint un niveau de population de $32 \times 10^{4} \mathrm{cfu} / \mathrm{g}$ de sol alors que les moins performantes ne dépassent pas $2 \times 10^{4} \mathrm{cfu} / \mathrm{g}$ de sol.

Tableau IV. Influence de la nature de l'échantillon de sol sur l'aptitude de souches de $F$ oxysporum non pathogènes à rétablir la résistance à $F$ oxysporum $\mathrm{fsp}$ lini (introduit à $3 \times 10^{3} \mathrm{cfu} / \mathrm{ml}$ de terre) dans l'échantillon de sol thermotraité (souches peu actives au cours d'un premier essai, voir fig 7). Pourcentage de plantes saines $70 \mathrm{j}$ après le semis.

$\left(^{*}\right)$ Les moyennes suivies d'une même lettre ne sont pas significativement différentes au seuil de $5 \%$ d'après le test de Duncan.

\begin{tabular}{|c|c|c|c|c|c|}
\hline \multirow{2}{*}{ Souches } & \multicolumn{4}{|c|}{ Sols (*) } & \multirow{2}{*}{$\begin{array}{c}\text { Ef fet } \\
\text { souches } \\
(*)\end{array}$} \\
\hline & Albenga & |Carmagnola| & Santena & Donnas & \\
\hline $\begin{array}{l}3 \\
11 v \\
22 \\
24 \\
7 \\
32 \\
--\end{array}$ & $\begin{array}{rr}27,5 & \mathrm{a}-\mathrm{c} \\
0,0 & \mathrm{~d} \\
35,7 & \mathrm{a}-\mathrm{b} \\
32,6 & \mathrm{a}-\mathrm{b} \\
1,3 & \mathrm{~d} \\
7,3 & \mathrm{c}-\mathrm{d} \\
0,0 & \mathrm{~d}\end{array}$ & $\begin{array}{rl}40,2 & \mathrm{a} \\
43,9 & \mathrm{a} \\
0,0 & \mathrm{~d} \\
0,0 & \mathrm{~d} \\
1,3 & \mathrm{~d} \\
1,4 & \mathrm{~d} \\
0,0 & \mathrm{~d}\end{array}$ & $\begin{array}{rr}3,5 & \mathrm{~d} \\
12,2 & \mathrm{~b}-\mathrm{d} \\
2,9 & \mathrm{~d} \\
0,0 & \mathrm{~d} \\
3,8 & \mathrm{~d} \\
5,2 & \mathrm{~d} \\
0,0 & \mathrm{~d}\end{array}$ & $\begin{array}{rr}9,6 & c-d \\
14,3 & c-d \\
1,4 & d \\
6,6 & c-d \\
0,0 & d \\
3,3 & d \\
0,0 & d\end{array}$ & $\begin{array}{r}20,2 \mathrm{a} \\
17,6 \mathrm{a}-\mathrm{b} \\
10,0 \mathrm{a}-\mathrm{c} \\
9,8 \mathrm{a}-\mathrm{c} \\
1,6 \mathrm{~b}-\mathrm{c} \\
4,3 \mathrm{a}-\mathrm{c} \\
0,0\end{array}$ \\
\hline $\begin{array}{l}\text { Effet } \\
\text { sols } \quad \text { (*) }\end{array}$ & 14,9 a & 12,4 a & 3,9 a & 5,1 a & \\
\hline
\end{tabular}


Tableau V. Influence de la nature de l'échantillon de sol sur l'aptitude de souches de $F$ oxysporum non pathogènes à rétablir la résistance à $F$ oxysporum $\mathrm{f} s p$ lini (introduit à $3 \times 10^{3} \mathrm{cfu} / \mathrm{ml}$ de terre) dans le sol thermotraité (souches très actives au cours d'un premier essai, voir fig 7). Pourcentage de plantes saines 83 j après le semis.

(") Les moyennes suivies d'une même lettre ne sont pas significativement différentes au seuil de $5 \%$ d'après le test de Duncan.

\begin{tabular}{|c|c|c|c|c|c|}
\hline \multirow{2}{*}{ Souches } & \multicolumn{4}{|c|}{ Sols (*) } & \multirow{2}{*}{$\begin{array}{c}\text { Effet } \\
\text { souches } \\
(*)\end{array}$} \\
\hline & Albenga & |Carmagnola & Santena & Donnas & \\
\hline $\begin{array}{l}8 a \\
26 s \\
6 a \\
21 a \\
23 a \\
17 c \\
22 s \\
20 a \\
9 a \\
8 s \\
--\end{array}$ & $\begin{array}{r}74,5 \mathrm{~h}-\mathrm{m} \\
88,5 \mathrm{a}-\mathrm{d} \\
85,5 \mathrm{a}-\mathrm{e} \\
64,7 \mathrm{c}-\mathrm{m} \\
92,9 \mathrm{a}-\mathrm{c} \\
46,0 \mathrm{i}-\mathrm{o} \\
32,6 \mathrm{~m}-\mathrm{p} \\
81,6 \mathrm{a}-\mathrm{e} \\
81,1 \mathrm{a}-\mathrm{f} \\
52,4 \mathrm{~h}-\mathrm{m} \\
8,5 \mathrm{p}-\mathrm{q}\end{array}$ & $\begin{array}{rr}45,8 & 1-\mathrm{o} \\
81,7 & \mathrm{a}-\mathrm{e} \\
80,7 & \mathrm{a}-\mathrm{h} \\
65,3 \mathrm{~d}-\mathrm{m} \\
55,7 & \mathrm{f}-\mathrm{n} \\
57,0 & \mathrm{e}-\mathrm{m} \\
43,6 & 1-\mathrm{o} \\
65,4 & \mathrm{~d}-\mathrm{m} \\
88,3 & \mathrm{a}-\mathrm{e} \\
70,1 & \mathrm{c}-\mathrm{m} \\
0,0 & \mathrm{q}\end{array}$ & $\begin{array}{rr}49,9 & \mathrm{~h}-\mathrm{n} \\
61,1 & \mathrm{~d}-\mathrm{m} \\
82,7 & \mathrm{a}-\mathrm{g} \\
49,8 & \mathrm{~g}-\mathrm{n} \\
86,1 \mathrm{a}-\mathrm{e} \\
57,7 \mathrm{e}-\mathrm{m} \\
14,8 \mathrm{p}-\mathrm{q} \\
87,8 \mathrm{a}-\mathrm{c} \\
73,0 \mathrm{~b}-1 \\
40,9 \text { 1-o } \\
0,0 & \mathrm{q}\end{array}$ & $\begin{array}{rr}95,9 & \mathrm{a}-\mathrm{b} \\
69,4 & \mathrm{c}-\mathrm{m} \\
83,1 & \mathrm{a}-\mathrm{f} \\
73,3 & \mathrm{a}-1 \\
97,1 & \mathrm{a} \\
60,3 & \mathrm{e}-\mathrm{m} \\
18,5 & \mathrm{n}-\mathrm{q} \\
94,1 & \mathrm{a}-\mathrm{c} \\
81,7 & \mathrm{a}-\mathrm{h} \\
41,3 & 1-\mathrm{o} \\
0,0 & \mathrm{q}\end{array}$ & $\begin{array}{rl}66,6 & \mathrm{a}-\mathrm{e} \\
75,8 & \mathrm{a}-\mathrm{d} \\
83,0 & \mathrm{a} \\
63,3 & \mathrm{~b}-\mathrm{e} \\
82,9 & \mathrm{a}-\mathrm{b} \\
55,1 & \mathrm{~d}-\mathrm{e} \\
27,4 & \mathrm{f} \\
82,2 & \mathrm{a}-\mathrm{b} \\
81,0 & \mathrm{a}-\mathrm{c} \\
51,2 & \mathrm{e}-\mathrm{f} \\
2,5 & \mathrm{~g}\end{array}$ \\
\hline $\begin{array}{l}\text { Effet } \\
\text { sols } \quad(*)\end{array}$ & $70,0 \mathrm{a}$ & 65,3 a & 59,1 a & $71,5 \mathrm{a}$ & \\
\hline
\end{tabular}

Les tableaux IV et $V$ présentent les pourcentages de plantes saines enregistrés $70 j$ et $83 j$ après le semis dans les sols précolonisés par les souches inefficaces et efficaces respectivement. Les résultats obtenus précédemment dans le sol d'Albenga sont confirmés globalement dans les 4 sols étudiés; l'activité des souches de $F$ oxysporum non pathogènes se manifeste de manière analogue dans les 4 sols considérés. Les 10 souches considérées comme efficaces (tableau V) permettent de limiter la maladie de manière importante, le pourcentage de plantes saines varie de 50 à $83 \%$, seule la souche 22 s présente un comportement analogue à celui des souches les moins efficaces (tableau IV).

\section{DISCUSSION}

Les résultats acquis au cours de cette expérimentation montrent que la méthode employée, initialement proposée par Alabouvette et al (1982), permet effectivement d'apprécier le niveau de réceptivité des sols aux fusarioses vasculaires. Les sols de Carmagnola et Santena se révèlent aussi résistants que le sol d'Albenga déjà connu pour sa résistance aux fusarioses de l'œillet et de la tomate (Garibaldi et al, 1983 ; Tamietti et Matta, 1984). Le sol de Donnas apparaît nettement moins résistant que les autres sols, sans toutefois présenter une sensibilité extrême lors du premier cycle cultural.

Dans tous les cas le traitement des sols à la vapeur qui détruit la plus grande partie de la microflore détruit également la résistance, indiquant que cette propriété repose fondamentalement sur des interactions microbiennes (Louvet et al, 1976 ; Scher et Baker, 1980 ; Tamietti et Matta, 1984 ; Tamietti et Alabouvette, 1986).

Les analyses microbiologiques réalisées montrent globalement que le sol le plus sensible est également celui qui présente les niveaux de populations microbiennes les plus faibles. Cependant, les résultats obtenus ne permettent pas de formuler une hypothèse précise quant au rôle respectif de la bactérioflore ou de la mycoflore dans les mécanismes de résistance. Compte tenu des résultats acquis précédemment (Garibaldi et al, 1985) et de l'importance de la population fusarienne, tant en valeur absolue qu'en valeur relative, il a été décidé de constituer une collection de souches de Fusarium spp isolés de ces 4 sols pour étudier leur aptitude à établir la résistance dans les sols traités à la chaleur.

La recolonisation du sol d'Albenga traité à la chaleur par les différentes souches de Fusarium a permis de montrer qu'une forte proportion de souches de $F$ oxysporum est capable d'établir la résistance à la fusariose du lin, alors qu'aucune souche de $F$ roseum n'appartient à la classe d'efficacité maximale (fig 2). Les souches de $F$ solani se répartissent quant à elles de manière uniforme dans les différentes classes de protection. Ces résultats permettent de penser que les $F$ oxysporum et, dans une moindre mesure, les $F$ solani jouent un rôle important dans les mécanismes de résistance des sols aux fusarioses vasculaires comme cela a déjà été signalé par Rouxel et al (1979) et Tamietti et Alabouvette (1986) respectivement pour les sols de Châteaurenard et de Noirmoutier.

Parmi les souches de $F$ oxysporum il existe une grande diversité quant à leur aptitude à induire la résistance dans un sol désinfecté. Cette diversité existe au sein des souches isolées d'un même sol, mais elle apparaît de façon beaucoup plus nette si l'on considère les souches isolées de sols différents. Ainsi le pourcentage de souches inactives est important parmi la population isolée du sol sensible de Donnas et le pourcentage de souches très efficaces est maximal pour les souches isolées du sol résistant d'Albenga. Ainsi ces résultats montrent que le niveau de résistance d'un sol dépend non seulement de la densité des populations de Fusarium mais aussi de la composition de ces populations de Fusarium, en particulier de F oxysporum.

L'aptitude des souches de $F$ oxysporum à induire la résistance dans des sols préalablement traités à la vapeur n'est pas corrélée avec leur aptitude à coloniser ces mêmes sols de manière plus ou moins intense. Ainsi, la souche 3 qui colonise les sols très intensément $\left(32 \times 10^{4}\right.$ 
cfu/g de sol) figure parmi les souches les moins efficaces (20\% de plantes saines). Au contraire, la souche 9 a qui est parmi les plus efficaces ( $81 \%$ de plantes saines) n'a colonisé le sol que de manière très limitée $\left(1 \times 10^{4} \mathrm{cfu} / \mathrm{g}\right.$ de sol).

De plus, dans ces conditions expérimentales, l'aptitude des souches à rendre résistants les échantillons de sols traités à la vapeur se manifeste de manière analogue dans les 4 types de sol étudiés. Le fait que les caractéristiques physico-chimiques des sols ne semblent pas modifier de façon déterminante le comportement des souches non pathogènes revêt une importance capitale en vue de l'utilisation de ces souches pour la lutte microbiologique contre les fusarioses vasculaires. A priori les souches efficaces isolées à partir d'un sol déterminé devraient être utilisables dans d'autres types de sol.

Ces résultats sont apparemment en contradiction avec les observations de Corman et al (1986) qui ont démontré l'existence d'une interaction sol-souche, à l'issue d'une expérimentation comparable à celle rapportée ici. Cependant l'influence du sol ne modifie pas de manière fondamentale le classement des souches de Fusarium spp en fonction de leur efficacité.

L'absence de corrélation entre l'aptitude des souches à coloniser les sols traités à la vapeur et leur aptitude à rendre ces sols résistants permet de penser que la résistance n'est pas basée sur un mécanisme d'antagonisme se déroulant dans le sol lui-même. II n'a d'ailleurs jamais été possible d'observer des relations d'antibiose ou d'hyperparasitisme entre souches pathogènes et non pathogènes de $F$ oxysporum. Si les mécanismes de résistance des sols reposent effectivement sur des relations de compétition entre souches pathogènes et non pathogènes de $F$ oxysporum, celles-ci se déroulent vraisemblablement dans la rhizosphère ou à la surface même de la racine, pour la conquête des sites d'infection (Schneider, 1984).

Cependant, certaines observations non présentées dans cet article suggèrent que la plante pourrait jouer un rôle direct dans les mécanismes de résistance induits par les $F$ oxysporum non pathogènes : ceux-ci induiraient des réactions de prémunition qui limiteraient l'invasion de ia plante par l'agent pathogène (Tamietti, non publié).

\section{REMERCIEMENTS}

Les auteurs remercient MM C Alabouvette et A Matta pour leur révision critique de cet article.

\section{RÉFÉRENCES}

Alabouvette C, Couteaudier Y, Louvet J (1982) Comparaison de la réceptivité de différents sols et substrats de culture aux fusarioses vasculaires. Agronomie 2, 1-6

Baker KF (1978) Biological control of Phytophthora cinnamomi. Proc Internatl Plant Prop Soc 28, $72-79$

Bouhot D (1975) Recherches sur l'écologie des champignons parasites dans le sol. VII. Quantification de la technique d'estimation du potentiel infectieux des sols, terreaux et substrats infestés par Pythium sp. Ann Phytopathol 7, 147-154

Corman A, Couteaudier Y, Zegerman M, Alabouvette C (1986) Réceptivité des sols aux fusarioses vasculaires : méthode statistique d'analyse des résultats. Agronomie 6, 751-757

Garibaldi A, Dalla Guda C, D'Aquila F (1983) Osservazioni su terreni repressivi nei confronti di Fusarium oxysporum f sp dianthi (Prill et Del) Snyd et Hans. Riv Ortoflorofrutt it 67, 251-259

Garibaldi A, Brunatti F, Allocchio A (1985) Terreni repressivi verso Fusarium oxysporum $\mathrm{f} s \mathrm{sp}$ dianthi : isolamento di microrganismi e loro attività antagonistica in vaso. La difesa delle piante 2, 101-106

Gerlagh M (1968) Introduction of Ophiobolus graminis into new polders and its decline. Neth Jour Plant Pathol 74, (Suppl 2), 1-97

Henis Y, Ghaffar A, Baker R (1978) Integrated control of Rhizoctonia solani damping-off of radish. Effect of successive planting, PCNB, and Trichoderma harzianum on pathogen and disease. Phytopathology $68,900-907$

King EO, Ward MK, Raney DE (1954) Two simple media for the demonstration of pyocyanin and fluorescin. J Lab Clin Med 44, 301

Ko W-h, Ho W-c (1983) Screening soils for suppressiveness to Rhizoctonia solani and Pythium splendens. Ann Phytopathol Soc Jpn 49, 1-9

Kloepper JW, Leong J, Teintze M, Schroth MN (1980) Enhanced plant growth by siderophores produced by plant growth-promoting rhizobacteria. Nature $286,885-886$

Komada H (1975) Development of a selective medium for quantitative isolation of Fusarium oxysporum from natural soil. Rev Plant Prot Res 8, 114-125

Louvet J, Rouxel F, Alabouvette C (1976) Recher-ches sur la résistance des sols aux maladies: I - Mise en évidence de la nature microbiologique de la résistance d'un sol au développement de la fusariose vasculaire du melon. Ann Phytopathol 8, 425426

Ogawa K, Komada H (1984) Biological control of Fusarium wilt of sweet potato by non-pathogenic Foxysporum. Ann Phytopathol Soc Jpn 50, 1-9

Rouxel F, Alabouvette C, Louvet J (1979) Recherches sur la résistance des sols aux maladies: IV - Mise en évidence du rôle des Fusarium autochtones dans la résistance d'un sol à la fusariose vasculaire du melon. Ann Phytopathol 11, 199-207

Scher MF, Baker R (1980) Mechanisms of biological control in a Fusarium suppressive soil. Phytopathology $70,412-417$ 
Scher MF, Baker R (1982) Effects of Pseudomonas putida and synthetic iron chelator on induction of soil suppressiveness to Fusarium wilt pathogens. Phytopathology 72, 1 567-1 573

Schneider RW (1984) Effect of non-pathogenic strains of Fusarium oxysporum on celery root. Infection by Fusarium oxysporum $f \mathrm{sp}$ apii and a novel use of the Lineweaver Burk double reciprocal plot technique. Phytopathology 14, 646-653

Tamietti G, Alabouvette C (1986) Résistance des sols aux maladies : XIII - Rôle des Fusarium oxyspo- rum non pathogènes dans les mécanismes de résistance d'un sol de Noirmoutier aux fusarioses vasculaires. Agronomie 6, 541-548

Tamietti G, Matta A (1984) Fusarium lycopersici suppressive properties in the soil of Albenga (Italy): preliminary observations. Proc 6th Congr Union Phytopathol Mediterr, Cairo, 129-131

Tello-Marquina JC, Alabouvette C (1984) Observation sur la persistance dans le sol des microconidies de Fusarium oxysporum. Agronomie 4, 885-890 